\title{
Temporal characteristics of evaporation trends and their attribution to meteorological drivers in Roorkee (India)
}

\author{
Digambar Singh ${ }^{1}$, A R Kumar ${ }^{1}$, Vikas Goyal ${ }^{1}$, Manohar Arora $^{1}$, and Nageswara Allaka ${ }^{1}$ \\ ${ }^{1}$ National Institute of Hydrology
}

May 12, 2020

\begin{abstract}
Pan evaporation is an important indicator of atmospheric evaporative demand, and its long-term variation is of much concern in studies of climate change. Estimation of evaporation is also important for water budgeting and yet is difficult to quantify because of the combined effects of four meteorological variables: net radiation, wind speed, atmospheric humidity, and air temperature. This work considered the temporal trends of pan evaporation and the meteorological variables that affect them for a station located in Roorkee (India). In this study, observed meteorological data at NIH observatory for the period from 1987 to 2018 was used for trend analysis of the data (rainfall, relative humidity, maximum temperature, minimum temperature, average temperature, wind speed and pan evaporation). Evaporation was also estimated using Penman method, Meyer method and other empirical equations, and compared with the observed evaporation values. Anomalies in the time series of meteorological variables were computed to find out the magnitude of rise or fall in the series. Pettitt-Mann-Whitney (PMW) test for detection of shift in the time series has been carried out, and the trend and shift in meteorological data is correlated with the same in evaporation. Based on this research, a number of conclusions are drawn: (1) minimum temperature and relative humidity have been increasing whereas maximum temperature and wind speed have been decreasing during the period 1987-2018, (2) pan evaporation series has not shown any significant trend, except during post-monsoon when it decreased, (3) significant change points (shifts) in the time series of temperature, relative humidity and wind speed may attribute the influence of fast urbanization and enhanced anthropogenic activities in Roorkee town after creation of Uttarakhand as a separate State in the year 2000 .
\end{abstract}

\section{Hosted file}

Evaporation Trends Manuscript.doc available at https://authorea.com/users/320885/articles/ 450300-temporal-characteristics-of-evaporation-trends-and-their-attribution-tometeorological-drivers-in-roorkee-india

\begin{tabular}{|l|r|r|r|r|r|r|r|r|r|r|}
\hline \multirow{2}{*}{ Parameter } & \multicolumn{2}{|c|}{$\begin{array}{c}\text { Winter } \\
\text { (JF) }\end{array}$} & \multicolumn{2}{|c|}{$\begin{array}{l}\text { Pre-monsoon } \\
\text { (MAM) }\end{array}$} & \multicolumn{2}{c|}{$\begin{array}{c}\text { Monsoon } \\
\text { (JJAS) }\end{array}$} & \multicolumn{2}{|c|}{$\begin{array}{c}\text { Post- monsoon } \\
\text { (OND) }\end{array}$} & \multicolumn{2}{|r|}{ Annual } \\
\cline { 2 - 12 } & Mean & SD & Mean & SD & Mean & SD & Mean & SD & Mean & SD \\
\hline Rainfall (mm) & 66.1 & 49.1 & 74.3 & 47.3 & 880.7 & 258.5 & 19.0 & 27.2 & 1040 & 249.4 \\
\hline T min $\left({ }^{\circ} \mathrm{C}\right)$ & 8.6 & 0.8 & 18.9 & 1.0 & 25.2 & 0.6 & 15.4 & 1.2 & 17.8 & 0.7 \\
\hline T max $\left({ }^{\circ} \mathrm{C}\right)$ & 26.8 & 1.4 & 38.4 & 1.1 & 37.5 & 1.7 & 32.0 & 2.7 & 34.1 & 1.3 \\
\hline T average $\left({ }^{\circ} \mathrm{C}\right)$ & 17.7 & 0.7 & 28.6 & 0.7 & 31.3 & 0.8 & 23.7 & 1.3 & 26.0 & 0.5 \\
\hline $\begin{array}{l}\text { Wind speed } \\
\text { (km/hr) }\end{array}$ & 1.2 & 0.6 & 2.0 & 1.0 & 1.6 & 0.8 & 0.7 & 0.4 & 1.5 & 0.7 \\
\hline $\begin{array}{l}\text { Relative } \\
\text { Humidity (\%) }\end{array}$ & 87.5 & 4.4 & 58.3 & 4.3 & 79.4 & 3.3 & 79.2 & 4.8 & 76.1 & 3.4 \\
\hline $\begin{array}{l}\text { Evaporation } \\
\text { (mm) }\end{array}$ & 112.1 & 16.5 & 459.2 & 60.8 & 451.9 & 70.1 & 125.6 & 16.8 & 1148.8 & 132.7 \\
\hline
\end{tabular}




\begin{tabular}{|c|r|r|r|r|r|r|}
\hline Month & \multicolumn{1}{|c|}{$\begin{array}{c}\text { Pan } \\
\text { evaporation } \\
(\mathrm{mm})\end{array}$} & $\begin{array}{l}\text { Open surface } \\
\text { evaporation }(0.7 * \text { pan } \\
\text { evaporation) }\end{array}$ & $\begin{array}{c}\text { Penman } \\
\text { method }\end{array}$ & $\begin{array}{c}\text { Meyer } \\
\text { Method }\end{array}$ & $\begin{array}{c}\text { Empirical } \\
\text { formula } \\
\text { (EI) }\end{array}$ & $\begin{array}{c}\text { Empirical } \\
\text { formula } \\
\text { (EII) }\end{array}$ \\
\hline Jan & 29.6 & 20.7 & 17.4 & 21.7 & 10.1 & 14.9 \\
\hline Feb & 52.6 & 36.9 & 36.0 & 44.0 & 22.2 & 30.2 \\
\hline Mar & 97.0 & 67.9 & 97.7 & 117.5 & 62.2 & 80.7 \\
\hline Apr & 165.6 & 116.0 & 218.9 & 261.8 & 140.8 & 179.9 \\
\hline May & 196.6 & 137.6 & 280.9 & 332.8 & 181.2 & 228.5 \\
\hline Jun & 163.0 & 114.1 & 197.1 & 233.6 & 127.0 & 160.2 \\
\hline Jul & 111.5 & 78.1 & 81.3 & 99.1 & 50.3 & 68.1 \\
\hline Aug & 90.4 & 63.3 & 65.4 & 80.9 & 40.6 & 55.6 \\
\hline Sep & 86.8 & 60.8 & 67.0 & 84.3 & 40.5 & 58.1 \\
\hline Oct & 80.2 & 56.1 & 77.5 & 101.2 & 44.3 & 69.7 \\
\hline Nov & 45.4 & 31.8 & 44.8 & 59.7 & 24.8 & 41.1 \\
\hline Dec & 29.7 & 20.8 & 21.5 & 28.3 & 12.0 & 19.4 \\
\hline
\end{tabular}

\begin{tabular}{|l|l|l|l|l|}
\hline $\begin{array}{l}\text { Performance } \\
\text { Parameters }\end{array}$ & $\begin{array}{l}\text { Penman } \\
\text { Method }\end{array}$ & $\begin{array}{l}\text { Meyer } \\
\text { Method }\end{array}$ & $\begin{array}{l}\text { Empirical equation } \\
\text { (E-I) }\end{array}$ & $\begin{array}{l}\text { Empirical equation } \\
\text { (E-II) }\end{array}$ \\
\hline $\begin{array}{l}\text { Percentage error } \\
\text { (monthly values) }\end{array}$ & 4.96 & 27.53 & -34.19 & -12.38 \\
\hline $\begin{array}{l}\text { Coefficient of } \\
\text { Determination }\left(\mathrm{R}^{2}\right)\end{array}$ & 0.88 & 0.87 & 0.84 & 0.86 \\
\hline
\end{tabular}

\begin{tabular}{|l|r|r|r|r|}
\hline \multicolumn{1}{|c|}{ Season } & $\begin{array}{c}\text { Penman } \\
\text { Method }\end{array}$ & $\begin{array}{c}\text { Meyer } \\
\text { Method }\end{array}$ & $\begin{array}{c}\text { Empirical } \\
\text { formula (EI) }\end{array}$ & $\begin{array}{c}\text { Empirical } \\
\text { formula (EII) }\end{array}$ \\
\hline Pre-monsoon & $\mathbf{3 0 . 1 2}$ & $\mathbf{5 5 . 0 8}$ & -16.32 & $\mathbf{6 . 5 1}$ \\
\hline Monsoon & -9.07 & 10.20 & -42.81 & -24.30 \\
\hline Post-monsoon & -2.57 & 28.07 & -45.03 & -11.80 \\
\hline Winter & -33.16 & -16.07 & -60.53 & -42.44 \\
\hline
\end{tabular}

\begin{tabular}{|l|l|l|l|l|l|l|l|l|l|}
\hline \multirow{2}{*}{ Parameter } & \multicolumn{4}{|l}{ Winter } & \multicolumn{4}{l|}{ Pre-monsoon } & \multicolumn{2}{l|}{ Monsoon } \\
\cline { 2 - 11 } & $\mathrm{S}$ & $\boldsymbol{\tau}$ & $\mathrm{P}$ & $\mathrm{S}$ & $\boldsymbol{\tau}$ & $\mathrm{P}$ & $\mathrm{S}$ & $\boldsymbol{\tau}$ & $\mathrm{P}$ \\
\hline Rainfall & -147.0 & -0.297 & $\mathbf{0 . 0 1 8}$ & -49.0 & -0.099 & 0.435 & -7.0 & -0.014 & 0.922 \\
\hline T min & 124.0 & 0.250 & $\mathbf{0 . 0 4 6}$ & 232 & 0.468 & $\mathbf{0 . 0 0 0}$ & 56.0 & 0.113 & 0.372 \\
\hline T max & -182.0 & -0.368 & $\mathbf{0 . 0 0 3}$ & -77.0 & -0.155 & 0.218 & -157.0 & -0.318 & $\mathbf{0 . 0 1 1}$ \\
\hline T average & -68.0 & -0.137 & 0.277 & 110.0 & 0.222 & 0.077 & -148.0 & -0.298 & $\mathbf{0 . 0 1 7}$ \\
\hline Wind speed & -260.0 & -0.524 & $<\mathbf{0 . 0 0 0 1}$ & -226.0 & -0.456 & $\mathbf{0 . 0 0}$ & -300.0 & -0.605 & $<\mathbf{0 . 0 0 0 1}$ \\
\hline Humidity & 306.0 & 0.617 & $<\mathbf{0 . 0 0 0 1}$ & 120.0 & 0.242 & 0.054 & 68.0 & 0.137 & 0.277 \\
\hline Evaporation & -78.0 & -0.157 & 0.212 & -54.0 & -0.109 & 0.39 & -98.0 & -0.198 & 0.116 \\
\hline
\end{tabular}

\begin{tabular}{|l|l|l|l|l|l|l|}
\hline \multirow{2}{*}{ Parameter } & \multicolumn{4}{|l|}{ Post-monsoon } & \multicolumn{4}{l|}{ Annual } \\
\cline { 2 - 7 } & $\mathrm{S}$ & $\boldsymbol{\tau}$ & $\mathrm{P}$ & $\mathrm{S}$ & $\boldsymbol{\tau}$ & $\mathrm{P}$ \\
\hline Rainfall & 36.00 & 0.075 & 0.566 & -10.0 & -0.016 & 0.884 \\
\hline T min & 158.00 & 0.319 & $\mathbf{0 . 0 1 1}$ & 176.0 & 0.355 & $\mathbf{0 . 0 0 5}$ \\
\hline T max & -198.0 & 0.402 & $\mathbf{0 . 0 0 1}$ & -194.0 & -0.391 & $\mathbf{0 . 0 0 2}$ \\
\hline T average & -130.0 & -0.262 & $\mathbf{0 . 0 3 6}$ & -114.0 & -0.230 & 0.067 \\
\hline Wind speed & -242.0 & -0.488 & $<\mathbf{0 . 0 0 0 1}$ & -270.0 & -0.544 & $<\mathbf{0 . 0 0 0 1}$ \\
\hline Humidity & 256.0 & 0.516 & $<\mathbf{0 . 0 0 0 1}$ & 200.0 & 0.403 & $\mathbf{0 . 0 0 1}$ \\
\hline Evaporation & -127.0 & -0.256 & $\mathbf{0 . 0 4 1}$ & -94.0 & -0.190 & 0.132 \\
\hline
\end{tabular}




\begin{tabular}{|c|c|c|c|c|c|c|c|c|c|c|}
\hline \multirow[t]{2}{*}{ Parameter } & \multicolumn{2}{|l|}{ Winter } & \multicolumn{2}{|c|}{ Pre-monsoon } & \multicolumn{2}{|c|}{ Monsoon } & \multicolumn{2}{|c|}{ Post-monsoon } & \multicolumn{2}{|l|}{ Annual } \\
\hline & $\begin{array}{l}\text { Sen's } \\
\text { Slope }\end{array}$ & $\begin{array}{l}\% \\
\text { Change }\end{array}$ & $\begin{array}{l}\text { Sen's } \\
\text { Slope }\end{array}$ & $\begin{array}{l}\% \% \\
\text { Change }\end{array}$ & $\begin{array}{l}\text { Sen's } \\
\text { Slope }\end{array}$ & $\begin{array}{l}\% \\
\text { Change }\end{array}$ & $\begin{array}{l}\text { Sen's } \\
\text { Slope }\end{array}$ & $\begin{array}{l}\% \\
\text { Change }\end{array}$ & $\begin{array}{l}\text { Sen's } \\
\text { Slope }\end{array}$ & $\begin{array}{l}\% \\
\text { Change }\end{array}$ \\
\hline Rainfall & -0.723 & -0.35 & -0.244 & -0.11 & -0.049 & 0 & 0.005 & 0.01 & 0.124 & 0 \\
\hline $\mathrm{T} \min$ & 0.03 & 0.11 & 0.066 & 0.11 & 0.018 & 0.02 & 0.053 & 0.11 & 0.039 & 0.07 \\
\hline $\mathrm{T} \max$ & -0.069 & -0.08 & -0.027 & -0.02 & -0.085 & -0.07 & -0.103 & -0.1 & -0.062 & -0.06 \\
\hline $\mathrm{T}$ average & -0.013 & -0.02 & 0.024 & 0.03 & -0.042 & -0.04 & -0.049 & -0.07 & -0.022 & -0.03 \\
\hline Wind speed & -0.045 & -1.2 & -0.065 & -1.04 & -0.072 & -1.44 & -0.028 & -1.28 & -0.057 & -1.22 \\
\hline Humidity & 0.378 & 0.14 & 0.15 & 0.08 & 0.083 & 0.03 & 0.36 & 0.15 & 0.226 & 0.1 \\
\hline Evaporation & -0.398 & -0.11 & -0.359 & -0.03 & -2.028 & -0.14 & -0.614 & -0.16 & -2.839 & -0.08 \\
\hline
\end{tabular}

\begin{tabular}{|l|l|l|l|l|l|}
\hline Parameter & Winter & Pre-monsoon & Monsoon & Post-monsoon & Annual \\
\hline Rainfall & - & No shift & No shift & No shift & No shift \\
\hline T min & No shift & + & + & + & + \\
\hline T max & No shift & No shift & No shift & - & - \\
\hline T average & No shift & No shift & No shift & No shift & No shift \\
\hline Wind speed & - & - & - & - & - \\
\hline umidity & + & No shift & No shift & + & + \\
\hline Evaporation & No shift & No shift & No shift & No shift & No shift \\
\hline
\end{tabular}

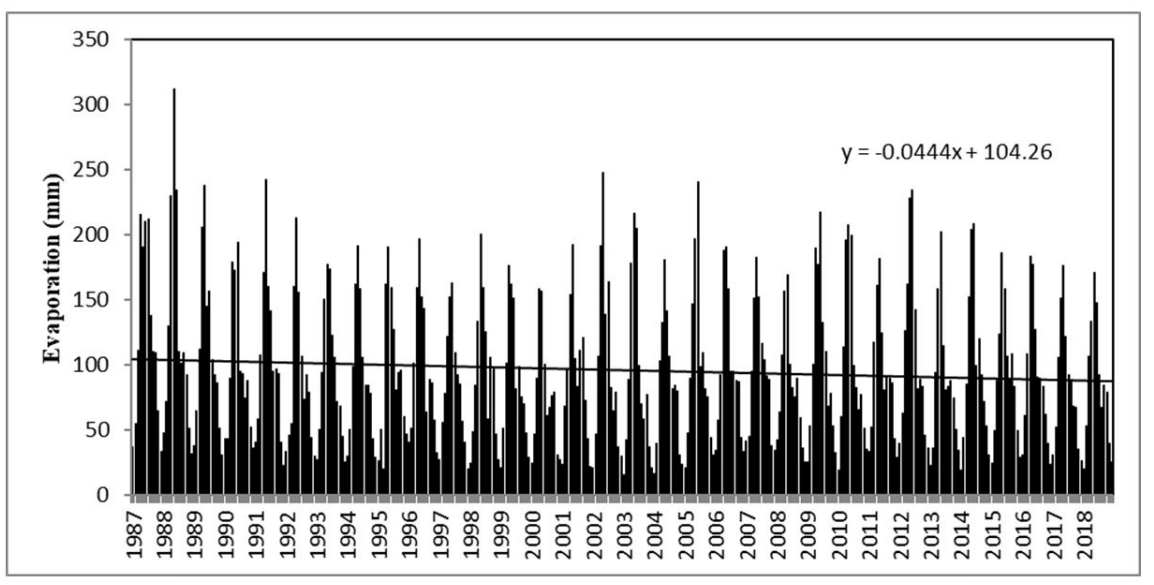



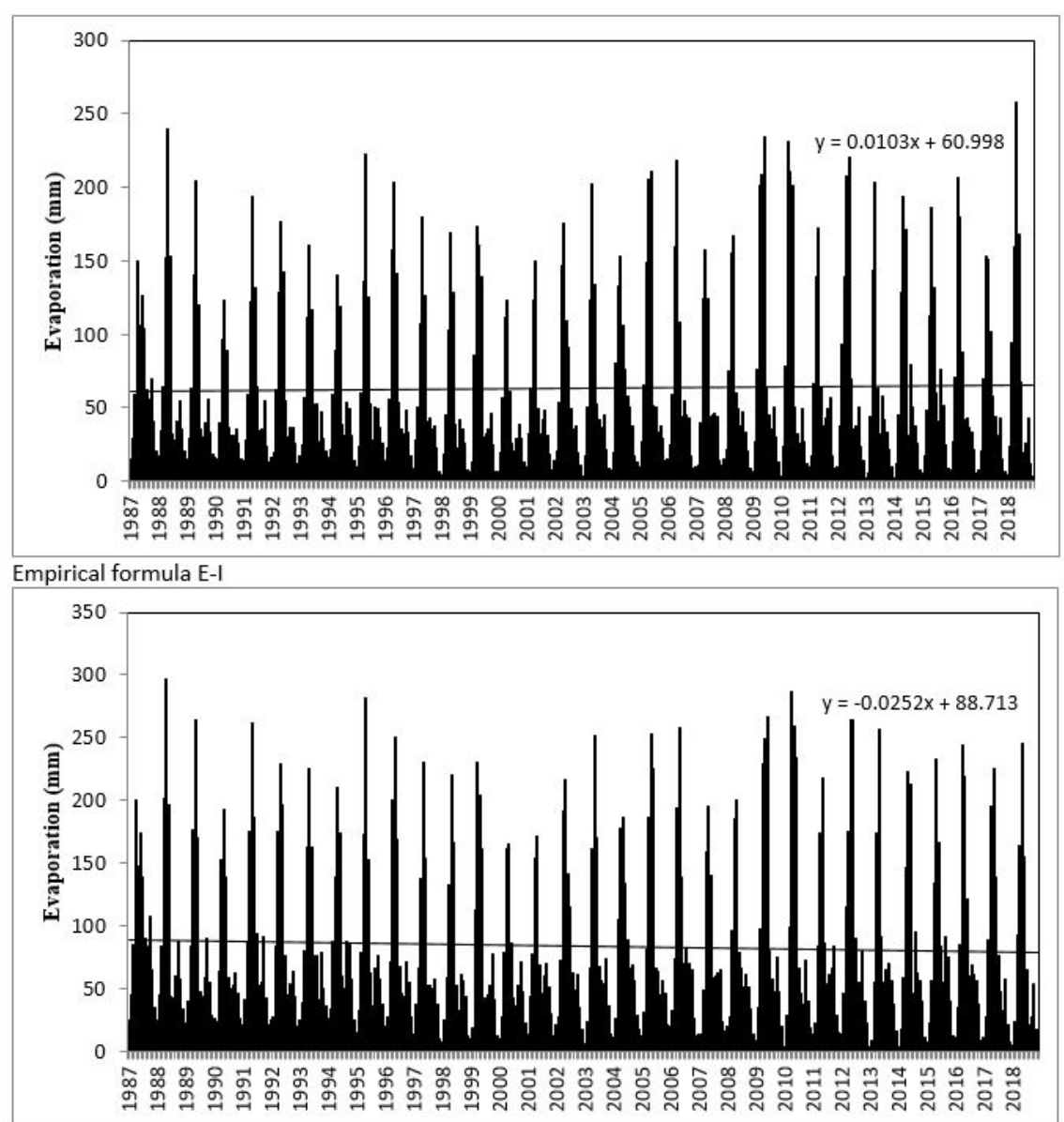

Empirical formula E-II 

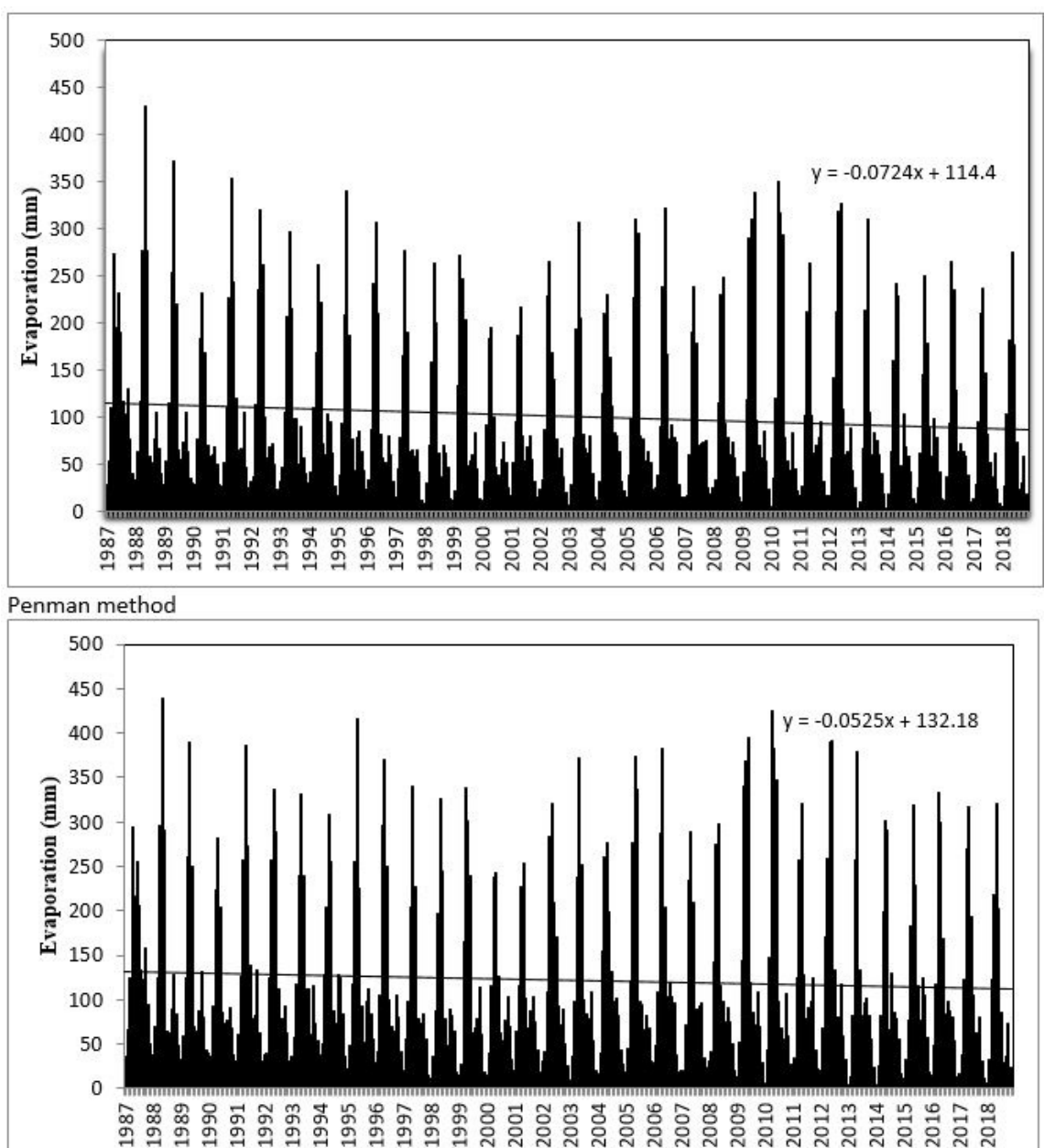

Meyer method 


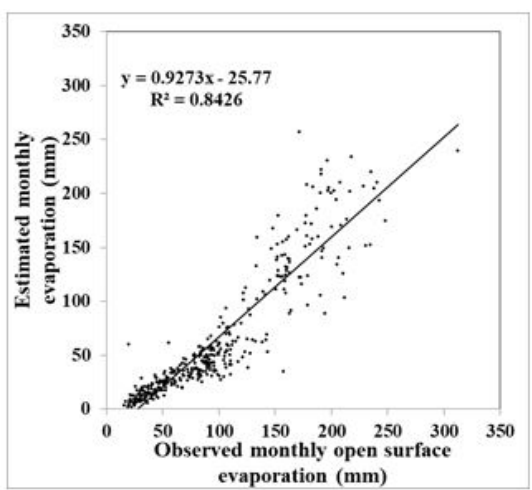

Empirical equation E-I

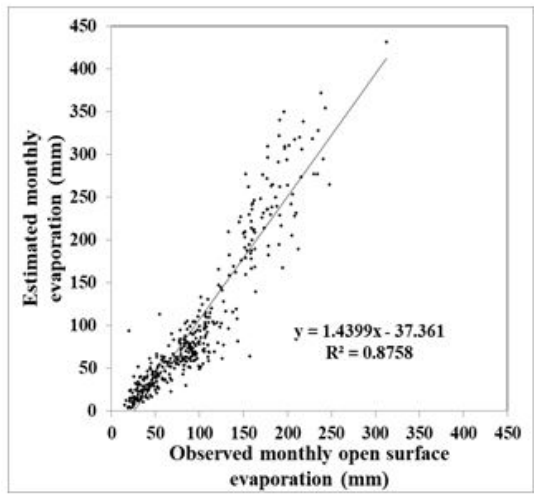

(ii) Penman method

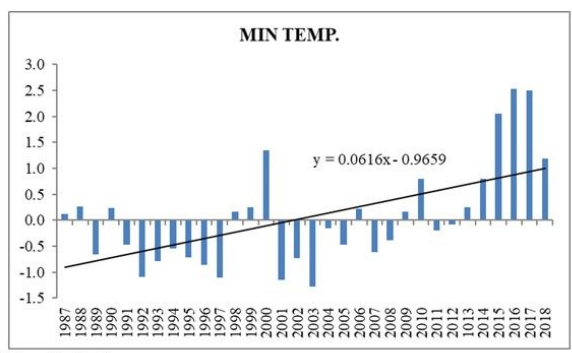

(i) Minimum temperature

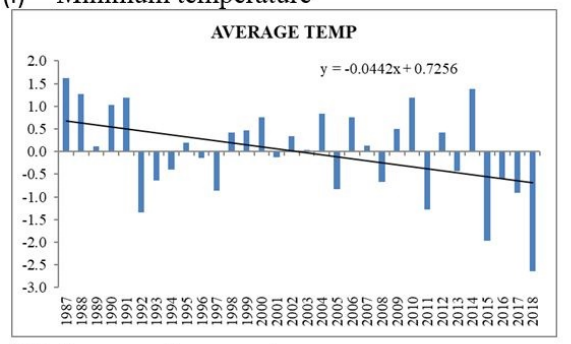

(iii) Average temperature

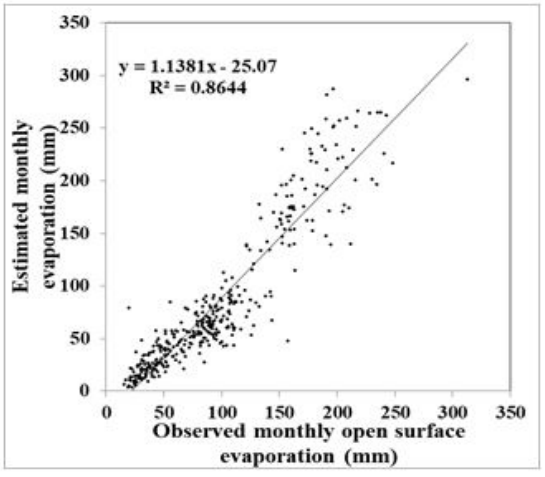

(i) Empirical equation E-II

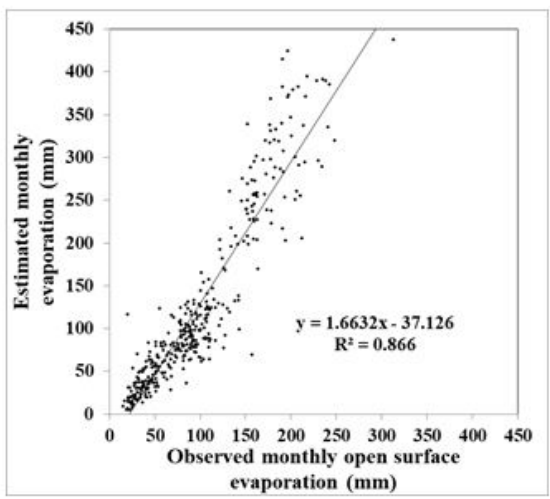

(iii) Meyer method

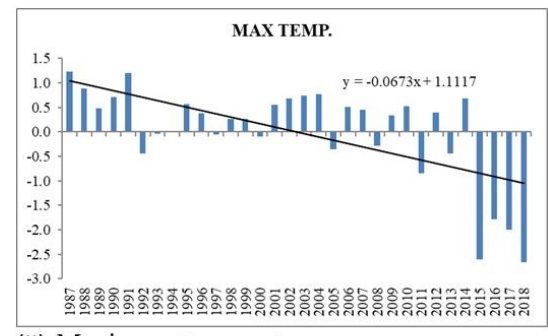

(ii) Maximum temperature

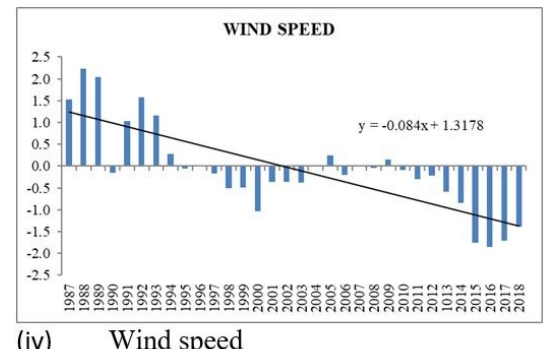




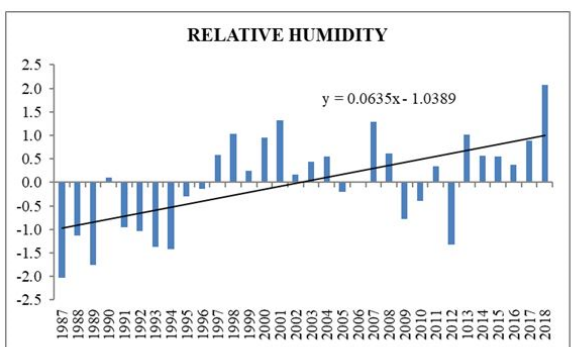

(v) Relative humidity

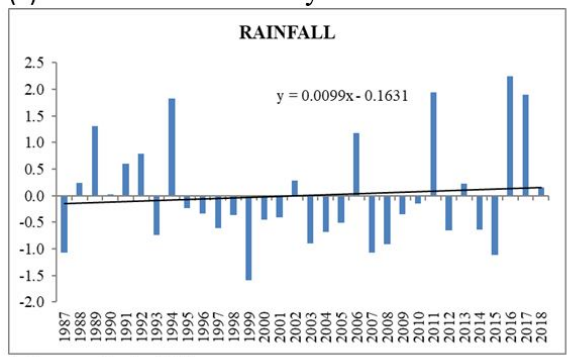

(vi) Rainfall
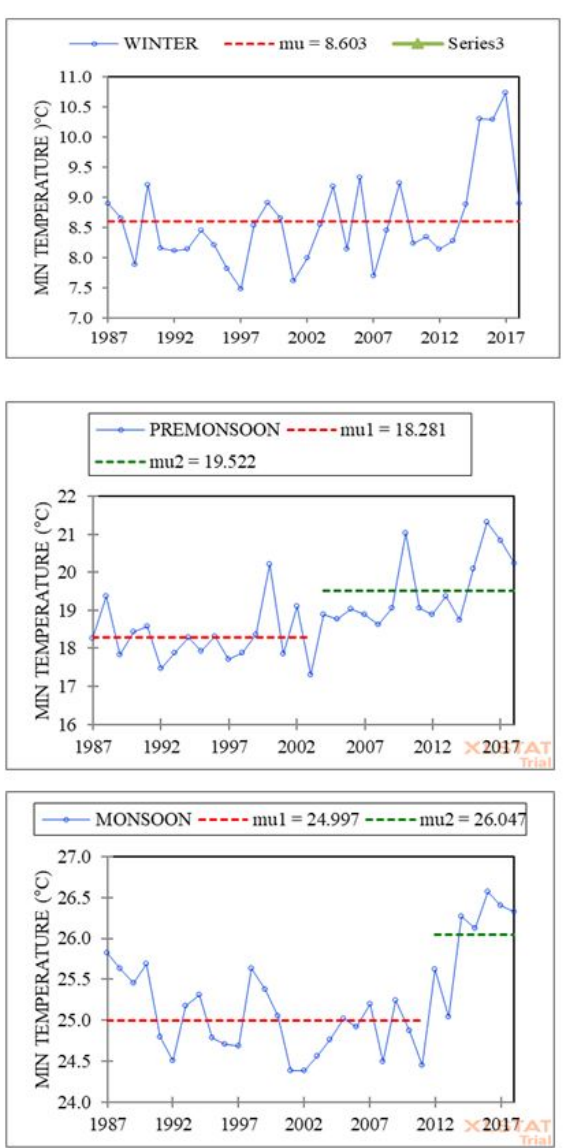

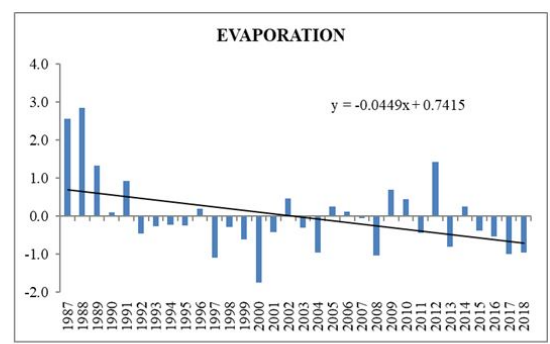

(iv) Evaporation
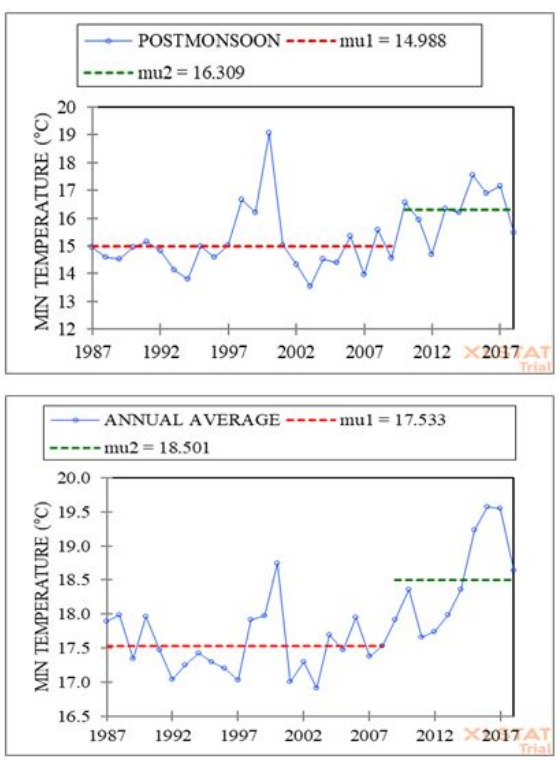

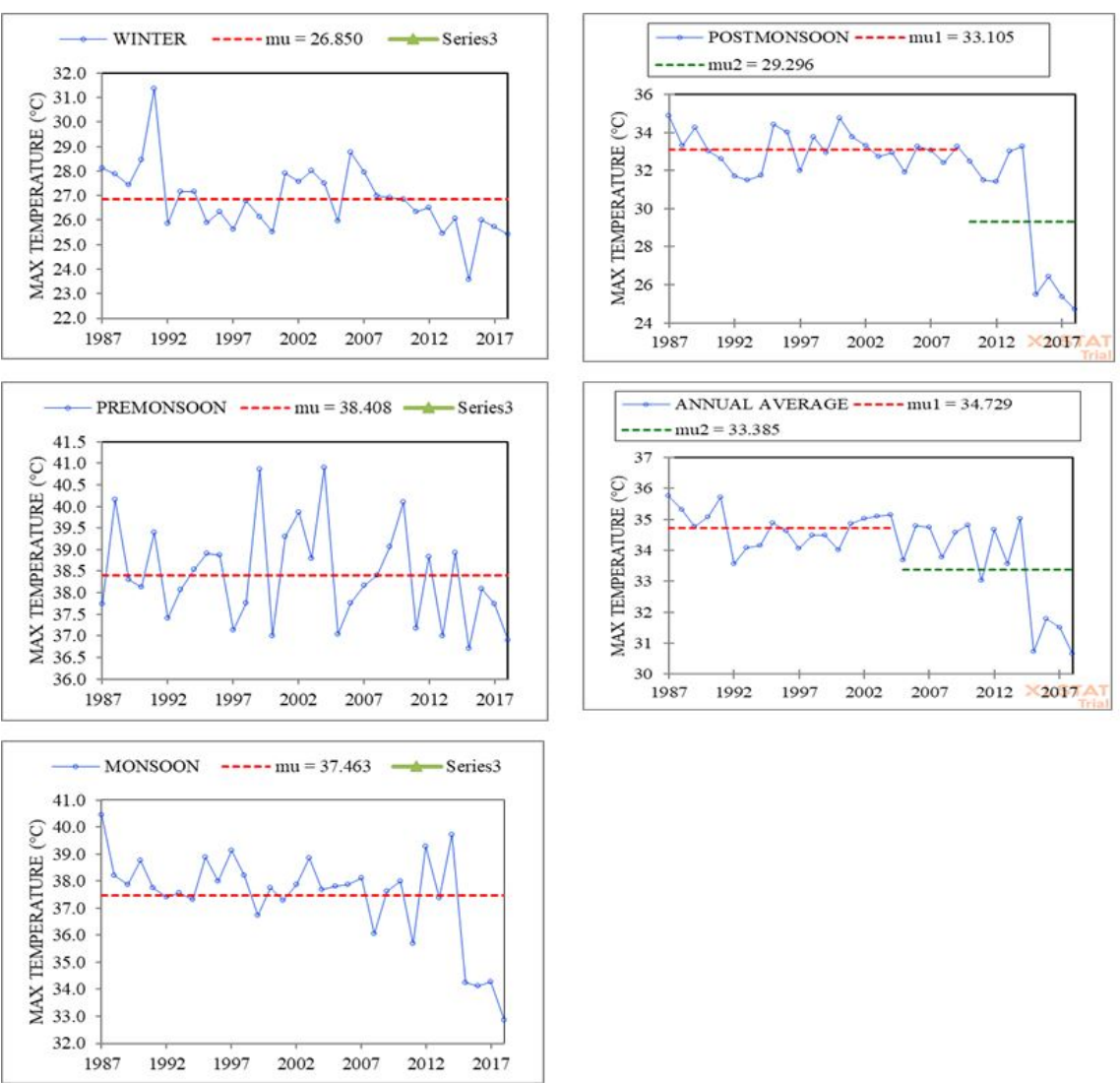

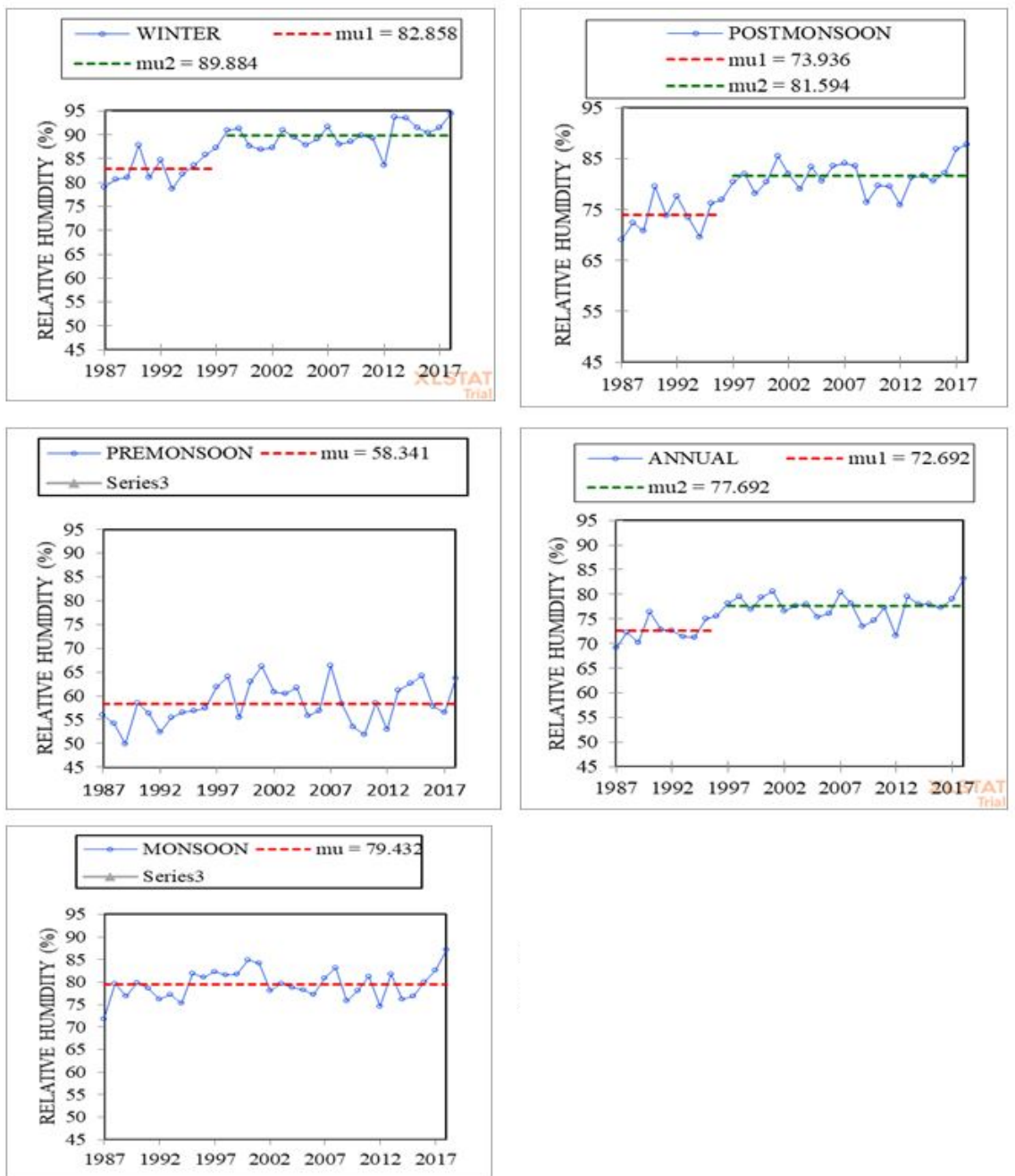

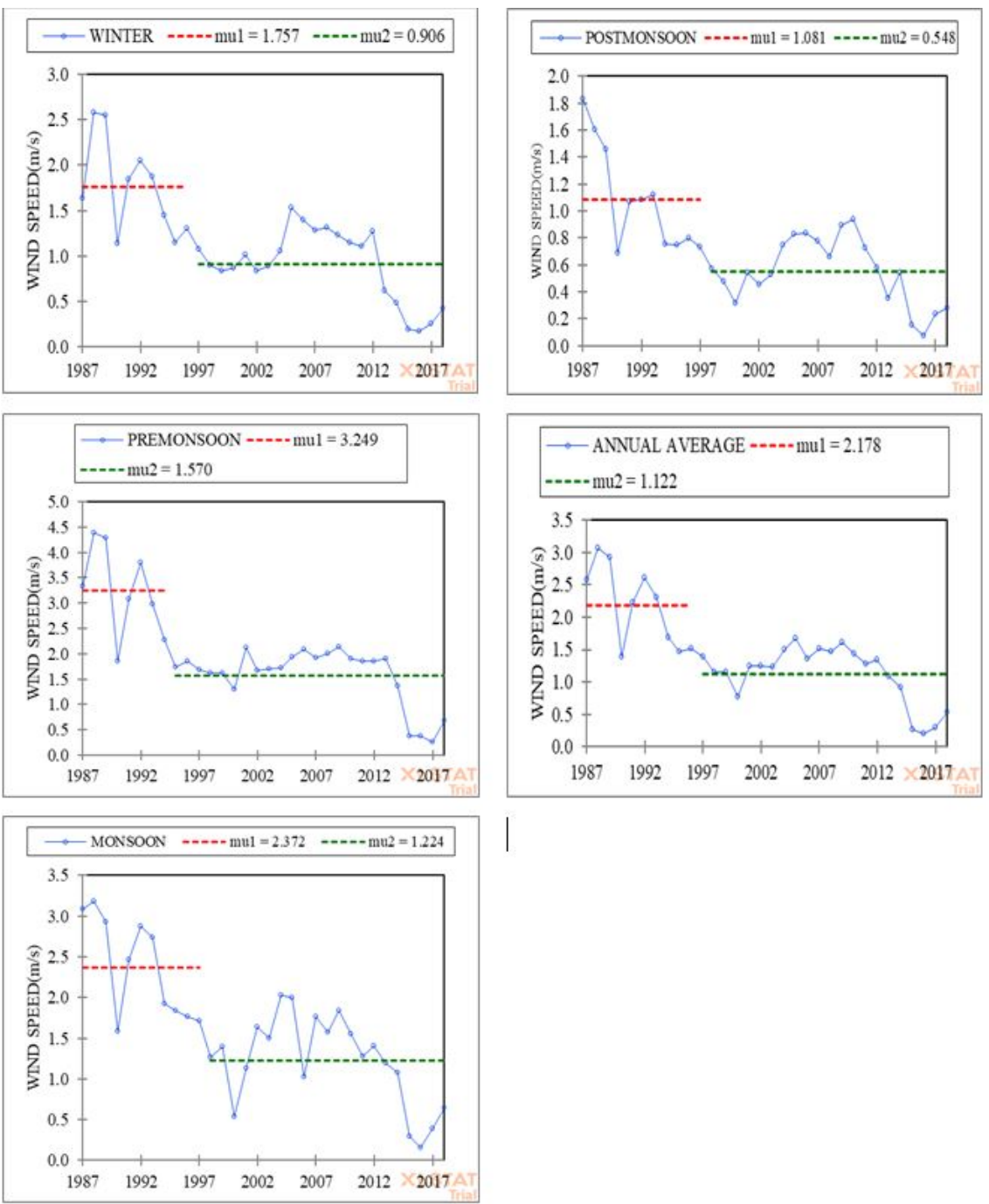

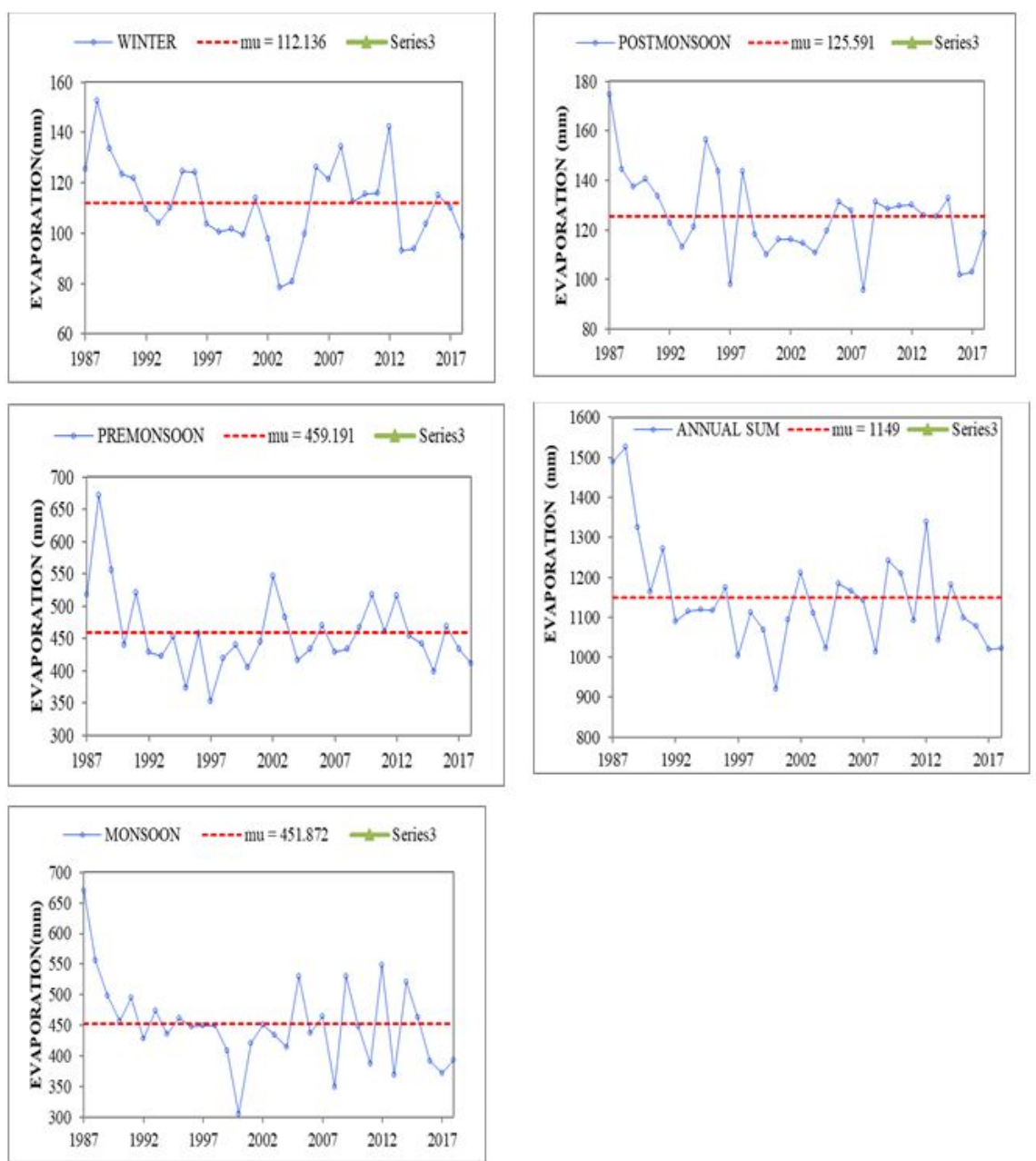